\title{
Upper bounds for the density of universality
}

\author{
by \\ JÖRn STEuding (Frankfurt)
}

1. Introduction and statement of results. The Riemann zeta-function is for $\operatorname{Re} s>1$ given by

$$
\zeta(s)=\prod_{p \text { prime }}\left(1-\frac{1}{p^{s}}\right)^{-1}=\sum_{n=1}^{\infty} \frac{1}{n^{s}},
$$

and by analytic continuation elsewhere, except for a simple pole at $s=1$. Voronin [11] proved a remarkable universality property of the Riemann zetafunction, namely that every continuous function $g(s)$, defined on the disc $\{s \in \mathbb{C}:|s| \leq r\}$ with some $r<1 / 4$, and analytic in the interior, can be approximated uniformly by shifts of the logarithm of the Riemann zetafunction in the strip $1 / 2<\operatorname{Re} s<1$.

Moreover, the set of translates $\log \zeta(3 / 4+s+i \tau)$ which approximate $g(s)$ with a given accuracy has even a positive lower density: if meas $A$ stands for the Lebesgue measure of the set $A$, then, for any $\varepsilon>0$,

$$
\liminf _{T \rightarrow \infty} \frac{1}{T} \text { meas }\left\{\tau \in[0, T]: \max _{|s| \leq r}\left|\log \zeta\left(\frac{3}{4}+s+i \tau\right)-g(s)\right|<\varepsilon\right\}>0 .
$$

Similar results were obtained for a large zoo of zeta-functions, for example, Dirichlet $L$-functions by Voronin [11], Dedekind zeta-functions by Reich [9], Hurwitz zeta-functions with certain parameters by Bagchi [1], Dirichlet series with multiplicative coefficients by Laurinčikas [6, §9.2], and RankinSelberg $L$-functions by Matsumoto [8].

The Linnik-Ibragimov conjecture states that all functions, given by a Dirichlet series, which are analytically continuable to the left of the halfplane of absolute convergence and satisfy additionally some natural growth conditions, are universal.

For more details on universality see [6] and [7].

2000 Mathematics Subject Classification: 11M06, 11M41.

Key words and phrases: universality, Riemann zeta-function, value distribution, Dirichlet series with periodic coefficients. 
However, the known proofs of universality theorems are ineffective, giving neither an estimate for the first approximating translate $\tau$ nor bounds for the positive lower density. The aim of this paper is to prove upper bounds for the upper density of universality.

Denote by $B_{r}$ the closed disc of radius $r>0$ with center at the origin. For a meromorphic function $L(s)$, an analytic function $g: B_{r} \rightarrow \mathbb{C}$ with fixed $r \in(0,1 / 4)$, and a positive $\varepsilon$ we define the densities

$$
\begin{aligned}
& \underline{d}(\varepsilon, g, L):=\liminf _{T \rightarrow \infty} \frac{1}{T} \operatorname{meas}\left\{\tau \in[0, T]: \max _{|s| \leq r}\left|L\left(\frac{3}{4}+s+i \tau\right)-g(s)\right|<\varepsilon\right\}, \\
& \bar{d}(\varepsilon, g, L):=\limsup _{T \rightarrow \infty} \frac{1}{T} \operatorname{meas}\left\{\tau \in[0, T]: \max _{|s| \leq r}\left|L\left(\frac{3}{4}+s+i \tau\right)-g(s)\right|<\varepsilon\right\} .
\end{aligned}
$$

For sufficiently large classes of functions $L(s)$ and $g(s)$, we will prove upper bounds for the upper density $\bar{d}(\varepsilon, g, L)$ which tend to 0 as $\varepsilon \rightarrow 0$. For that purpose we consider analytic isomorphisms $g: B_{r} \rightarrow B_{1}$, i.e. the inverse $g^{-1}$ exists and is analytic. Obviously, such a function $g$ has exactly one simple zero $\varrho$ in the interior of $B_{r}$; moreover, it turns out (by the Schwarz lemma, see $[5, \S$ VII.2]) that

$$
g(s)=r \exp (i \phi) \frac{\varrho-s}{r^{2}-\bar{\varrho} s} \quad \text { with } \phi \in \mathbb{R},|\varrho|<r .
$$

Denote by $\mathcal{A}_{r}$ the class of analytic isomorphisms from $B_{r}$ (with fixed $0<$ $r<1 / 4)$ to the unit disc. Further, let $N\left(\sigma_{1}, \sigma_{2}, T ; L\right)$ count the number of zeros of $L(s)$ in $1 / 2<\sigma_{1}<\operatorname{Re} s<\sigma_{2}<1,0 \leq \operatorname{Im} s<T$ (according multiplicities). Our main result is

Theorem 1. Suppose that $g \in \mathcal{A}_{r}$. Assume that $L(s)$ is analytic in $\operatorname{Re} s \geq 3 / 4-r$ except for at most o(T) many singularities inside $\operatorname{Re} s \geq$ $3 / 4-r, 0 \leq \operatorname{Im} s \leq T$ as $T \rightarrow \infty$, and that $\underline{d}(\varepsilon, g, L)>0$ for all $\varepsilon>0$. Then, for any $\varepsilon \in\left(0,(2 r)^{-1}(1 / 4+\operatorname{Re}|\varrho|)\right)$,

$$
\begin{aligned}
\bar{d}(\varepsilon, g, L) \leq & \frac{8 r^{3} \varepsilon}{r^{2}-|\varrho|^{2}} \\
& \times \limsup _{T \rightarrow \infty} \frac{1}{T} N\left(\frac{3}{4}+\operatorname{Re} \varrho-2 r \varepsilon, \frac{3}{4}+\operatorname{Re} \varrho+2 r \varepsilon, T ; L\right) .
\end{aligned}
$$

This theorem relates the density of universality to the value distribution of $L$. Note that one can obtain similar estimates for other $c$-values instead of $c=0$ whenever $c$ lies in the interior of $B_{r}$. Since too many well distributed zeros of $L(s)$ in $1 / 2<\operatorname{Re} s<1$ violate the universality property, it seems very likely that the limit in (2) exists in general.

Applying this to $L(s)=\log \zeta(s)$, we deduce 
Corollary 2. Let $g \in \mathcal{A}_{r}$. Then, for any $\varepsilon \in\left(0,(2 r)^{-1}(1 / 4+\operatorname{Re}|\varrho|)\right)$,

$$
\begin{aligned}
\bar{d}(\varepsilon, \exp g, \zeta(s)) \leq & \frac{8 e r^{3} \varepsilon}{r^{2}-|\varrho|^{2}} \\
& \times \lim _{T \rightarrow \infty} \frac{1}{T} N\left(\frac{3}{4}+\operatorname{Re} \varrho-2 r \varepsilon, \frac{3}{4}+\operatorname{Re} \varrho+2 r \varepsilon, T ; \log \zeta(s)\right) \\
= & o(\varepsilon) .
\end{aligned}
$$

Therefore, the decay of $\bar{d}(\varepsilon, f, \zeta)$ as $\varepsilon \rightarrow 0$ is more than linear in $\varepsilon$.

For a further application we consider Dirichlet series with periodic coefficients

$$
L(s, f):=\sum_{n=1}^{\infty} \frac{f(n)}{n^{s}}, \quad \text { where } \quad f(n+q)=f(n)
$$

for all $n \in \mathbb{N}$ and some $q \in \mathbb{N}$. Obviously, such series converge absolutely in Re $s>1$. By the periodicity of $f$,

$$
L(s, f)=\frac{1}{q^{s}} \sum_{a=1}^{q} f(a) \zeta\left(s, \frac{a}{q}\right), \quad \text { where } \quad \zeta(s, x):=\sum_{n=0}^{\infty} \frac{1}{(n+x)^{s}}
$$

for fixed $0<x \leq 1$ and $\operatorname{Re} s>1$ is the Hurwitz zeta-function. In view of well known properties of Hurwitz zeta-functions, it turns out that $L(s, f)$ is analytically continuable throughout $\mathbb{C}$ with at most one simple pole at $s=1$. As an immediate consequence of the joint universality of Dirichlet $L$-functions we obtain

THEOREM 3. Suppose that $q$ is an odd prime, $f$ is not a multiple of a character mod $q$, and $f(q)=0$. Let $g(s)$ be a continuous function on $B_{r}$ which is analytic in the interior of $B_{r}$. Then, for any $\varepsilon>0$,

$$
\underline{d}(\varepsilon, g, L(s, f))>0 \text {. }
$$

For example, the Davenport-Heilbronn zeta-function, given by

$$
\frac{1-i \kappa}{2} L(s, \chi)+\frac{1+i \kappa}{2} L(s, \bar{\chi}), \quad \text { where } \quad \kappa:=\frac{\sqrt{10-2 \sqrt{5}}-2}{\sqrt{5}-1}
$$

and $\chi$ is the character mod 5 with $\chi(2)=i$, satisfies the conditions of the universality theorem above.

With regard to Theorem 1 we get

Corollary 4. Suppose that $q$ is an odd prime, $f$ is not a multiple of a character mod $q$ satisfying $f(1)=1, f(q)=0$, and $g \in \mathcal{A}_{r}$. Then, for any $\varepsilon \in\left(0,(2 r)^{-1}(1 / 4+\operatorname{Re}|\varrho|)\right)$ and any $\sigma_{1} \in(1 / 2,3 / 4+\operatorname{Re} \varrho-2 r \varepsilon)$,

$$
\begin{aligned}
\bar{d}(\varepsilon, g, L(s, f)) \leq & \frac{8 r^{3} \varepsilon}{4 \pi\left(r^{2}-|\varrho|^{2}\right)\left(3 / 4+\operatorname{Re} \varrho-2 r \varepsilon-\sigma_{1}\right)} \\
& \times \log \left(\frac{1}{q^{2 \sigma_{1}}} \sum_{a=1}^{q}|f(a)|^{2} \zeta\left(2 \sigma_{1}, \frac{a}{q}\right)\right) .
\end{aligned}
$$


We conclude with one more example. Consider for an odd prime $q$,

$$
L\left(s, 1_{q}\right)=\frac{1}{q^{s}} \zeta\left(s, \frac{1}{q}\right), \quad \text { where } \quad 1_{q}(n):= \begin{cases}1 & \text { if } n \equiv 1 \bmod q, \\ 0 & \text { if } n \neq \equiv 1 \bmod q .\end{cases}
$$

By Theorem $3, L\left(s, 1_{q}\right)$ has the universality property, and, using Corollary 4 , we obtain after some calculations, for sufficiently small $\varepsilon>0$,

$$
\bar{d}\left(\varepsilon, g, L\left(s, 1_{q}\right)\right) \leq \frac{2 \zeta(9 / 8+|\varrho| / 2) r^{3} \varepsilon}{q^{9 / 8+|\varrho| / 2} \pi\left(r^{2}-|\varrho|^{2}\right)},
$$

which tends to zero as $q \rightarrow \infty$.

\section{Proofs of Theorem 1 and Corollary 2}

Proof of Theorem 1. The idea of the proof is that the zero $\varrho$ of $g$ is related to some zeros of $L(s)$ in $1 / 2<\operatorname{Re} s<1$. Since $g$ maps the boundary of $B_{r}$ onto the unit circle, Rouché's theorem (see [5, §VI.1]) implies the existence of one simple zero $\lambda$ of $L(z)$ in

$$
K_{\tau}:=\left\{z=3 / 4+s+i \tau: s \in B_{r}\right\}
$$

whenever

$$
\max _{s \in B_{r}}|L(3 / 4+s+i \tau)-g(s)|<\varepsilon<1=\max _{s \in B_{r}}|g(s)| .
$$

We may say that the zero $\varrho$ of $g(s)$ generates such a zero $\lambda$ of $L(s)$.

Universality is a phenomenon that appears in intervals. We prove an upper bound for the distance of different translates generating the same zero $\lambda$ of $L(s)$ :

Lemma 5. Suppose that a zero $\lambda$ of $L(s)$, generated by $\varrho$, lies in two different translates $K_{\tau_{1}}$ and $K_{\tau_{2}}$. Then

$$
\left|\tau_{1}-\tau_{2}\right|<\frac{8 r^{4} \varepsilon}{r^{2}-|\varrho|^{2}} .
$$

Proof. Suppose that there exist

$$
s_{j}=\operatorname{Re} \lambda-3 / 4+i t_{j} \in B_{r} \quad \text { and } \quad \tau_{j} \in \mathbb{R} \quad \text { with } \quad L\left(3 / 4+s_{j}+i \tau_{j}\right)=0,
$$
for $j=1,2$, such that

$$
\lambda=3 / 4+s_{1}+i \tau_{1}=3 / 4+s_{2}+i \tau_{2} .
$$

In view of (1),

$$
\left|g\left(s_{2}\right)-g\left(s_{1}\right)\right|=r \frac{r^{2}-|\varrho|^{2}}{\left|r^{2}-\bar{\varrho} s_{1}\right| \cdot\left|r^{2}-\bar{\varrho} s_{2}\right|}\left|s_{2}-s_{1}\right| .
$$

We deduce from (3) that $\left|g\left(s_{j}\right)\right|<\varepsilon$ for $j=1,2$, and therefore

$$
\left|\tau_{1}-\tau_{2}\right|=\left|t_{2}-t_{1}\right| \leq \frac{4 r^{3}}{r^{2}-|\varrho|^{2}}\left|g\left(s_{2}\right)-g\left(s_{1}\right)\right|<\frac{8 r^{3} \varepsilon}{r^{2}-|\varrho|^{2}},
$$

which proves the lemma. 
We continue with the proof of the theorem. Denote by $\mathcal{I}_{j}(T)$ the disjoint intervals in $[0, T]$ such that (3) is valid exactly for

$$
\tau \in \bigcup_{j} \mathcal{I}_{j}(T)=: \mathcal{I}(T) .
$$

Using Lemma 5 , in every interval $\mathcal{I}_{j}(T)$ lie at least

$$
1+\left[\frac{r^{2}-|\varrho|^{2}}{8 r^{3} \varepsilon} \operatorname{meas} \mathcal{I}_{j}(T)\right] \geq \frac{r^{2}-|\varrho|^{2}}{8 r^{3} \varepsilon} \operatorname{meas} \mathcal{I}_{j}(T)
$$

zeros $\lambda$ of $L(s)$ in the strip $1 / 2<\operatorname{Re} s<1$; here $[x]$ stands for the greatest integer $\leq x$. Therefore, the number $\mathcal{N}(T)$ of such zeros $\lambda$ satisfies the estimate

$$
\frac{8 r^{3} \varepsilon}{r^{2}-|\varrho|^{2}} \mathcal{N}(T) \geq \operatorname{meas} \mathcal{I}(T)
$$

The next step is to replace $\mathcal{N}(T)$ by the zero counting function appearing in the theorem.

Obviously, the value distribution of $L(z)$ in $K_{\tau}$ is ruled by that of $g(s)$ in $B_{r}$. This gives a restriction on the real parts of zeros $\lambda$.

Lemma 6. Let $\lambda$ be a zero of $L(s)$ generated by $\varrho$. Then

$$
|\operatorname{Re} \lambda-3 / 4-\operatorname{Re} \varrho|<2 r \varepsilon \text {. }
$$

Proof. Let $s \in B_{r}$. If $|g(s)| \geq \varepsilon$, then, in view of (3),

$$
|L(3 / 4+s+i \tau)| \geq|g(s)|-|g(s)-L(3 / 4+s+i \tau)|>0 .
$$

Since (1) implies $|g(s)| \geq|\varrho-s| /(2 r)$, we obtain the estimate of the lemma by taking the real parts.

From Lemma 6 we find that

$$
\mathcal{N}(T) \leq N(3 / 4+\operatorname{Re} \varrho-2 r \varepsilon, 3 / 4+\operatorname{Re} \varrho+2 r \varepsilon, T ; L) .
$$

On the other hand, since $\underline{d}(\varepsilon, g, L)>0$, there exists an increasing sequence $\left(T_{k}\right)$ with $\lim _{k \rightarrow \infty} T_{k}=\infty$ such that for any $\delta>0$,

$$
\operatorname{meas} \mathcal{I}\left(T_{k}\right) \geq(\bar{d}(\varepsilon, g, L)-\delta) T_{k} .
$$

Consequently, this together with (5) and (4) leads to

$$
\frac{8 r^{3} \varepsilon}{r^{2}-|\varrho|^{2}} N\left(\frac{3}{4}+\operatorname{Re} \varrho-2 r \varepsilon, \frac{3}{4}+\operatorname{Re} \varrho+2 r \varepsilon, T_{k} ; L\right) \geq(\bar{d}(\varepsilon, g, L)-\delta) T_{k} .
$$

Sending $\delta \rightarrow 0$ yields the estimate (2) of the theorem. Since the set of singularities of $L(s)$ in $\sigma \geq 3 / 4-r$ has zero density but $\underline{d}(\varepsilon, g, L)>0$, the singularities do not affect the above observations. The theorem is proved.

Proof of Corollary 2. We apply Theorem 1 to $L(s)=\log \zeta(s)$. Bohr and Landau proved that the number of zeros of $\zeta(s)$ in $\operatorname{Re} s>\sigma, 0<\operatorname{Im} s \leq T$ 
is $o(T)$ for any fixed $\sigma>1 / 2$ (see $[10, \S 9.17])$; hence, the set of singularities of $\log \zeta(s)$ has density zero. As shown by Bohr and Jessen, the limit

$$
\lim _{T \rightarrow \infty} \frac{1}{T} N\left(\frac{3}{4}+\operatorname{Re} \varrho-\delta, \frac{3}{4}+\operatorname{Re} \varrho+\delta, T ; \log \zeta\right)
$$

exists, and tends to 0 as $\varepsilon \rightarrow 0$ (see [2, Hilfssatz 6]). Further, one has

$$
\begin{aligned}
\max _{s \in B_{r}} \mid \zeta(3 / 4 & +s+i \tau)-\exp g(s) \mid \\
& \leq \max _{s \in B_{r}}|\exp g(s)| \cdot \max _{s \in B_{r}}|\exp (\log \zeta(3 / 4+s+i \tau)-g(s))-1| \\
& \leq e \max _{s \in B_{r}}|\zeta(3 / 4+s+i \tau)-\exp g(s)| .
\end{aligned}
$$

The corollary is shown.

\section{Proofs of Theorem 3 and Corollary 4}

Proof of Theorem 3. Denote by $\varphi$ Euler's totient. For the $\varphi(q)$ pairwise inequivalent characters $\chi_{j} \bmod q$ define the matrix

$$
\Xi=\left(\chi_{j}(n)\right)_{1 \leq n, j \leq \varphi(q)} \text {. }
$$

By the properties of characters to a prime modulus the matrix $\Xi$ is invertible (since pairwise inequivalent characters are linearly independent over $\mathbb{C}$, and $\operatorname{det} \Xi$ is a Vandermonde determinant). Hence there exist uniquely determined complex numbers $c_{1}, \ldots, c_{\varphi(q)}$ such that

$$
f(a)=\sum_{j=1}^{\varphi(q)} c_{j} \chi_{j}(a) \quad \text { for } a=1, \ldots, \varphi(q),
$$

where at least two distinct coefficients $c_{j}$ are non-vanishing (since $f$ is not a multiple of a character $\bmod q$ ). In view of $f(q)=0$ we obtain the representation

$$
L(s, f)=\sum_{j=1}^{\varphi(q)} c_{j} L\left(s, \chi_{j}\right) .
$$

Put $l_{g}:=1+\sup _{s \in B_{r}}|g(s)|$. Then $g(s)+l_{g} \neq 0$ for $s \in B_{r}$. Without loss of generality we may assume that $c_{1}, c_{2} \neq 0$. Now define

$$
g_{\chi_{1}}(s)=\frac{g(s)+l_{g}}{c_{1}}, \quad g_{\chi_{2}}=-\frac{l_{g}}{c_{2}} \quad \text { and } \quad g_{\chi_{j}}(s)=\eta \quad \text { for } j=3, \ldots, \varphi(q),
$$

where $\eta$ is a small positive parameter, to be chosen later. Using the joint universality of Dirichlet $L$-functions [1], for any $\delta>0$ we obtain

$$
\liminf _{T \rightarrow \infty} \frac{1}{T} \operatorname{meas}\left(\tau \in[0, T]: \max _{1 \leq j \leq \varphi(q)} \max _{s \in B_{r}}\left|L\left(s+i \tau, \chi_{j}\right)-g_{\chi_{j}}(s)\right|<\delta\right)>0 .
$$

Consequently, (6) implies 


$$
\begin{aligned}
\max _{s \in B_{r}}|L(s+i \tau, f)-g(s)| & \leq \sum_{j=1}^{\varphi(q)}\left|c_{j}\right| \max _{s \in B_{r}}\left|L\left(s+i \tau, \chi_{j}\right)-g_{\chi_{j}}(s)\right|+\eta \sum_{j=3}^{\varphi(q)}\left|c_{j}\right| \\
& \leq \delta(q-1) \max _{1 \leq j \leq q-1}\left|c_{j}\right|+\eta(q-3) .
\end{aligned}
$$

For sufficiently small $\delta$ and $\eta$ the assertion of the theorem follows.

Proof of Corollary 4. Denote by $N(\sigma, T)$ the number of zeros of $L(s, f)$ in the region $\operatorname{Re} s>\sigma, 0<\operatorname{Im} s \leq T$. Then Littlewood's lemma (see [10, $\S 9.9])$ states that

$$
\int_{\sigma_{1}}^{\sigma_{2}} N(\sigma, T) d \sigma=\frac{1}{2 \pi i} \int_{\mathcal{R}} \log L(s, f) d s+O(1)
$$

where $\mathcal{R}$ is the rectangular contour with vertices $\sigma_{1}, \sigma_{2}, \sigma_{1}+i T, \sigma_{2}+i T$ with $1 / 2<\sigma_{1}<1<\sigma_{2}$, and where the error term arises from the possible pole of $L(s, f)$ at $s=1$ (to define here $\log L(s, f)$ we choose the principal branch of the logarithm on the real axis whereas for other points $s$ the value of the logarithm is obtained by analytic continuation). Obviously, we may choose $\sigma_{2}$ such that $L(s, f)$ has no zeros in the half-plane $\operatorname{Re} s \geq \sigma_{2}$. Since $f(1)=1$ a standard application of Jensen's formula (see [5, §IX.1] and [3]) shows that the right hand side of (7) equals

$$
\begin{aligned}
\frac{1}{2 \pi} \int_{0}^{T} \log \left|L\left(\sigma_{1}+i t, f\right)\right| d t & +O(\log T) \\
& \leq \frac{T}{4 \pi} \log \left(\frac{1}{T} \int_{0}^{T}\left|L\left(\sigma_{1}+i t\right)\right|^{2} d t\right)+O(\log T) .
\end{aligned}
$$

Therefore, using the mean square formula [4]

$$
\lim _{T \rightarrow \infty} \frac{1}{T} \int_{0}^{T}|L(\sigma+i t, f)|^{2} d t=\frac{1}{q^{2 \sigma}} \sum_{a=1}^{q}|f(a)|^{2} \zeta\left(2 \sigma, \frac{a}{q}\right)
$$

valid for $\sigma>1 / 2$, we may replace (7) by

$$
\sum_{\substack{\operatorname{Re} \lambda>\sigma_{1} \\ 0<\operatorname{Im} \lambda \leq T}}\left(\operatorname{Re} \lambda-\sigma_{1}\right) \leq \frac{T}{4 \pi} \log \left(\frac{1}{q^{2 \sigma}} \sum_{a=1}^{q}|f(a)|^{2} \zeta\left(2 \sigma, \frac{a}{q}\right)\right)+O(\log T)
$$

as $T \rightarrow \infty$; here the sum on the left hand side is taken over all zeros $\lambda$ of $L(s, f)$, not necessarily generated by $\varrho$. Since, for $1 / 2<\sigma_{1}<\sigma_{3}$,

$$
N\left(\sigma_{3}, T\right) \leq \frac{1}{\sigma_{3}-\sigma_{1}} \sum_{\substack{\operatorname{Re} \lambda>\sigma_{1} \\ 0<\operatorname{Im} \lambda \leq T}}\left(\operatorname{Re} \lambda-\sigma_{1}\right)
$$

we may estimate 


$$
\begin{aligned}
& N(3 / 4+\operatorname{Re} \varrho-2 r \varepsilon, 3 / 4+\operatorname{Re} \varrho+2 r \varepsilon, T ; L(s, f)) \\
& \leq \frac{T}{4 \pi\left(3 / 4+\operatorname{Re} \varrho-2 r \varepsilon-\sigma_{1}\right)} \log \left(\frac{1}{q^{2 \sigma_{1}}} \sum_{a=1}^{q}|f(a)|^{2} \zeta\left(2 \sigma_{1}, \frac{a}{q}\right)\right)+O(\log T) .
\end{aligned}
$$

This leads in view of (2) to the estimate in the corollary.

Acknowledgements. The author thanks Prof. Laurinčikas and Prof. Garunkštis, both Vilnius University, and Prof. Matsumoto, Nagoya University, for several fruitful discussions on this topic as well as pointing out errors in a former version of the paper. Furthermore, the author is grateful for the kind hospitality at the Department of Number Theory and Probability Theory at Vilnius University during his visits. Last but not least, the author thanks the referee for his valuable remarks.

\section{References}

[1] B. Bagchi, The statistical behaviour and universality properties of the Riemann zetafunction and other allied Dirichlet series, Ph.D. thesis, Indian Statistical Institute, Calcutta, 1981.

[2] H. Bohr und B. Jessen, Über die Werteverteilung der Riemannschen Zetafunktion, Acta Math. 58 (1932), 1-55.

[3] R. Garunkštis and J. Steuding, On the zero distributions of Lerch zeta-functions, Analysis (Munich) 22 (2002), 1-12.

[4] A. Kačenas and A. Laurinčikas, On the periodic zeta-function, Liet. Mat. Rink. 41 (2001), 214-226 (in Russian).

[5] S. Lang, Complex Analysis, 2nd ed., Springer, 1985.

[6] A. Laurinčikas, Limit Theorems for the Riemann Zeta-Function, Kluwer, Dordrecht, 1996.

[7] K. Matsumoto, Probabilistic distribution theory for the value of zeta-function, Sūgaku 53 (2001), 279-296 (in Japanese).

[8] - The mean values and the universality of Rankin-Selberg L-functions, in: Number Theory, Proc. Turku Sympos. on Number Theory in memory of Kustaa Inkeri (1999), M. Jutila and T. Metsänkylä (eds.), de Gruyter, Berlin, 2001, 201-221.

[9] A. Reich, Wertverteilung von Zetafunktionen, Arch. Math. (Basel) 34 (1980), 440451.

[10] E. C. Titchmarsh, The Theory of the Riemann Zeta-Function, 2nd ed., revised by D. R. Heath-Brown, Oxford Univ. Press, 1986.

[11] S. M. Voronin, A theorem on the "universality" of the Riemann zeta-function, Izv. Akad. Nauk SSSR Ser. Mat. 39 (1975), 475-486 (in Russian); English transl.: Math. USSR-Izv. 9 (1975), 443-453.

Institut für Algebra und Geometrie

Johann Wolfgang Goethe-Universität Frankfurt

Robert-Mayer-Str. 10

60054 Frankfurt, Germany

E-mail: steuding@math.uni-frankfurt.de 